\title{
Crohn's Disease with Mesalazine Allergy that Was Difficult to Differentiate from Comorbid Ulcerative Colitis
}

\author{
Rumiko Tsuboi, Satohiro Matsumoto, Hiroyuki Miyatani and Hirosato Mashima
}

\begin{abstract}
:
An 18-year-old man diagnosed with ileocolonic Crohn's disease with circumferential strictures of the ascending colon started treatment with mesalazine and subsequently underwent right hemicolectomy. After surgery, the patient was started on adalimumab, and the clinical course was favorable. Nine months postoperatively, colonoscopy revealed granular mucosa with circumferential and continuous involvement from the transverse colon down to the rectum, findings which resembled ulcerative colitis. Mesalazine allergy was suspected, and the inflammatory findings resolved after discontinuing mesalazine. In patients of inflammatory bowel disease receiving mesalazine with an atypical clinical course, the possibility of mesalazine allergy must be borne in mind.
\end{abstract}

Key words: mesalazine allergy, Crohn's disease, ulcerative colitis

(Intern Med 58: 649-654, 2019)

(DOI: 10.2169/internalmedicine.1607-18)

\section{Introduction}

Mesalazine is the mainstay of pharmacologic treatment for inflammatory bowel disease, including ulcerative colitis and Crohn's disease. However, this drug can sometimes exacerbate symptoms, such as a fever, diarrhea, and bloody stool. Some patients show intolerance to mesalazine, with manifestations that are often difficult to differentiate from those of exacerbation of the underlying inflammatory bowel disease $(1,2)$. While mesalazine has been reported to cause diarrhea in $4.6 \%$ of patients and bloody stool and a fever in $1.4 \%$ of patients with ulcerative colitis (1), the prevalence of mesalazine intolerance in patients with Crohn's disease remains unknown.

We encountered a patient with Crohn's disease with mesalazine allergy in whom the condition was difficult to differentiate from comorbid ulcerative colitis because the colonoscopic findings resembled those of ulcerative colitis.

\section{Case Report}

An 18-year-old man without any history of diseases or allergies visited a neighborhood clinic with a 3-month history of abdominal pain, diarrhea, and bloody stool. Intestinal regulators were prescribed, but the symptoms persisted. Colonoscopy revealed erosions, longitudinal ulcers, and a cobblestone appearance in the mucosa of the ileocecum and ascending colon, so Crohn's disease was suspected. The patient was admitted to our hospital for further management. On admission, the Crohn's disease activity index (CDAI) was determined to be 243 , the serum C-reactive protein (CRP) level was $8.98 \mathrm{mg} / \mathrm{dL}$, and the serum albumin level was $3.4 \mathrm{mg} / \mathrm{dL}$.

Colonoscopy performed at our hospital revealed longitudinal ulcers and circumferential strictures blocking the advance of the scope into the ascending colon; however, the mucosa extending from the transverse colon downward towards the anus was normal (Fig. 1a-f). A fluoroscopic examination revealed stenosis in the ascending colon measuring over $10 \mathrm{~cm}$ in length (Fig. 1g). Non-necrotizing granulomas were not found on a tissue biopsy. Neither computed tomography nor magnetic resonance enterography revealed any evidence of inflammation at any other part of the bowel than the ileocecum and ascending colon. Upper endoscopy showed no abnormal findings. Given these findings as well as those reported from the previous clinic, we made a diagnosis of ileocolonic Crohn's disease. 

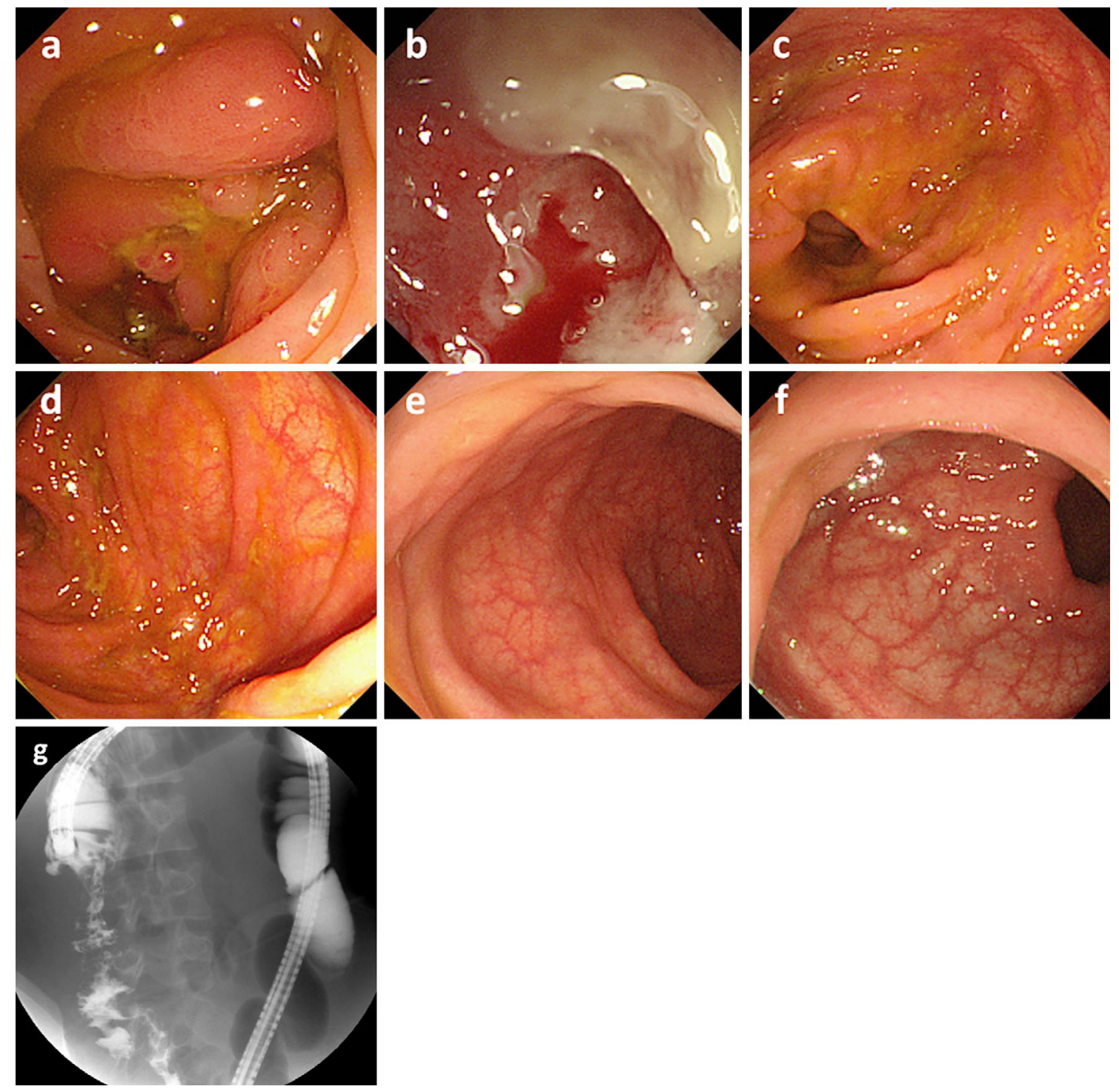

Figure 1. Colonoscopic findings on admission. a, b: Longitudinal ulcers and circumferential strictures blocking the advance of the scope were observed in the ascending colon. $\mathrm{c}$ : Transverse colon, $\mathrm{d}$ : Descending colon, e: Sigmoid colon, f: The rectal mucosa was normal. g: A fluoroscopic examination revealed stenosis in the ascending colon measuring over $10 \mathrm{~cm}$ in length.

As no relief of the circumferential strictures of the ascending colon could be expected from conservative medical treatment, we decided to introduce adalimumab as reset therapy after performing laparoscopic right hemicolectomy. While waiting for surgery, the patient was started on oral administration of time-dependent-release mesalazine at $3 \mathrm{~g} / \mathrm{d}$ and an enteral elemental diet. A histopathological examination of the resected surgical specimen revealed longitudinal ulcers extending from the ascending colon to the ileocecum, inflammatory cell infiltration in all the bowel mucosal layers, and non-necrotizing granulomas in the mucosal and submucosal layers (Fig. 2), features that were consistent with the diagnosis of Crohn's disease. After surgery, the patient was started on subcutaneous adalimumab. By 2 months after the treatment initiation, the CDAI had improved to 5 , and the serum CRP level had decreased to $0.05 \mathrm{mg} / \mathrm{dL}$.

However, 9 months after the surgery, the serum CRP level increased to $1.05 \mathrm{mg} / \mathrm{dL}$, and repeat colonoscopy was performed (Fig. 3). No abnormalities were observed in the terminal ileum or at the bowel anastomosis site, and the Rutgeerts score was i,0. However, granular mucosa was found, with circumferential and continuous involvement of the colon extending from the transverse colon to the rectum, resembling the endoscopic features of ulcerative colitis. While the Mayo Endoscopic subscore was 1, a biopsy revealed crypt abscesses. Ulcerative colitis of the total colitis type was therefore suspected. In accordance with the diagnosis of ulcerative colitis, the dose of mesalazine was increased to 4 g/d. Six months later, colonoscopy revealed slight aggravation of the inflammatory findings of the large bowel, with the patient complaining of persistent diarrhea. Therefore, the time-dependent-release mesalazine was switched to $\mathrm{pH}$ dependent-release mesalazine at $3.6 \mathrm{~g} / \mathrm{d}$.

Six months after this change in treatment, colonoscopy 

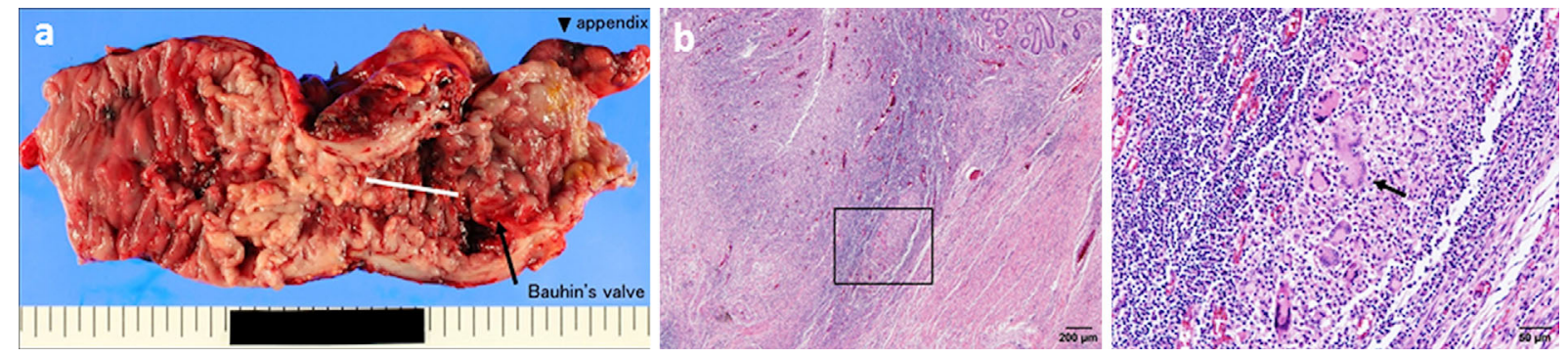

Figure 2. Surgical specimen. a: Macroscopic image of the surgical specimen. Longitudinal ulcers were observed in the segment of the bowel extending from the ascending colon to the ileocecum. The ascending colon was hard in consistency and shortened because of inflammation. b: Hematoxylin and Eosin (H\&E) staining, low magnification. Inflammatory cell infiltration was observed in all mucosal layers. c: H\&E staining, high magnification. Non-necrotizing granulomas (arrow) were observed in the mucosal and submucosal layers.
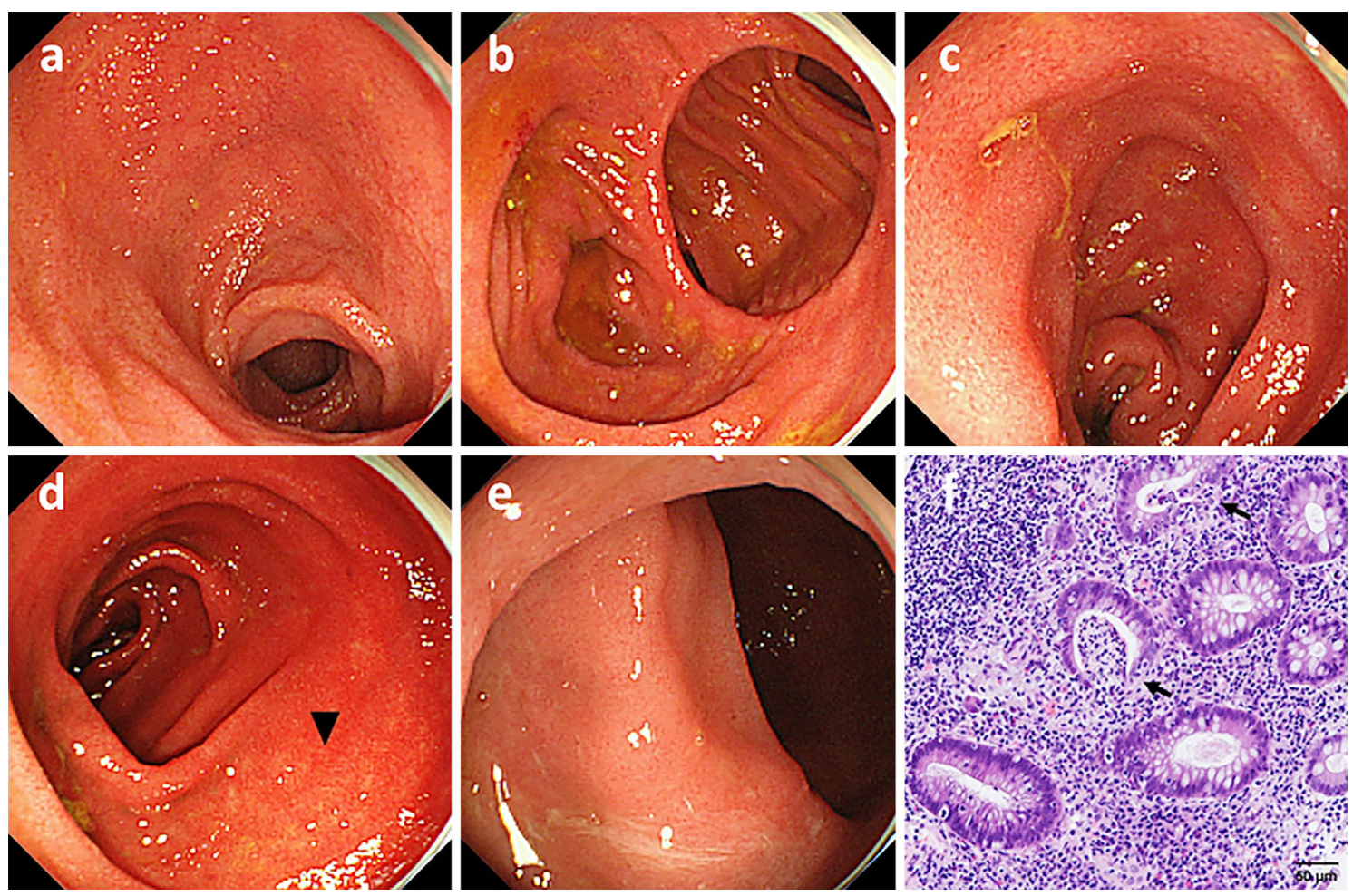

Figure 3. Colonoscopic findings at nine months after surgery. No abnormalities were observed in the terminal ileum (a) or at the postoperative bowel anastomotic site (b). Granularity of the mucosa was observed with circumferential and continuous bowel involvement, resembling the endoscopic features of ulcerative colitis of total colitis type (c: Descending colon. d: Sigmoid colon. e: Rectum). f: Histopathological findings of the biopsy specimen. Hematoxylin and Eosin staining, high magnification. Crypt abscesses (arrow) are seen.

still showed no marked improvement in the findings of the large bowel, and the Mayo Endoscopic subscore was 1. In addition, erosions were observed at the terminal ileum and at the bowel anastomotic site, which were diagnosed as representing diffuse aphthous ileitis, and the Rutgeerts score was i,3. Although backwash colitis could not be completely denied, we considered exacerbation of Crohn's disease (Fig. 4). The dose of adalimumab was increased to $40 \mathrm{mg} /$ wk. Mesalazine was switched again to the time-dependentrelease type at $4 \mathrm{~g} / \mathrm{d}$ doubling as treatment for $\mathrm{UC}$, and the enteral elemental diet was resumed. Six months later, colonoscopy revealed slight improvement in the inflammatory findings, and the Rutgeerts score was i,2. In contrast, the CDAI was 109, and the serum CRP level was $1.96 \mathrm{mg} /$ dL. Thus, the serum CRP level remained high, with persistent diarrhea.

None of the treatments attempted-increasing the dose of mesalazine, changing the type of mesalazine preparation used, and increasing the dose of adalimumab under suspicion of ulcerative colitis or inflammatory bowel disease un- 


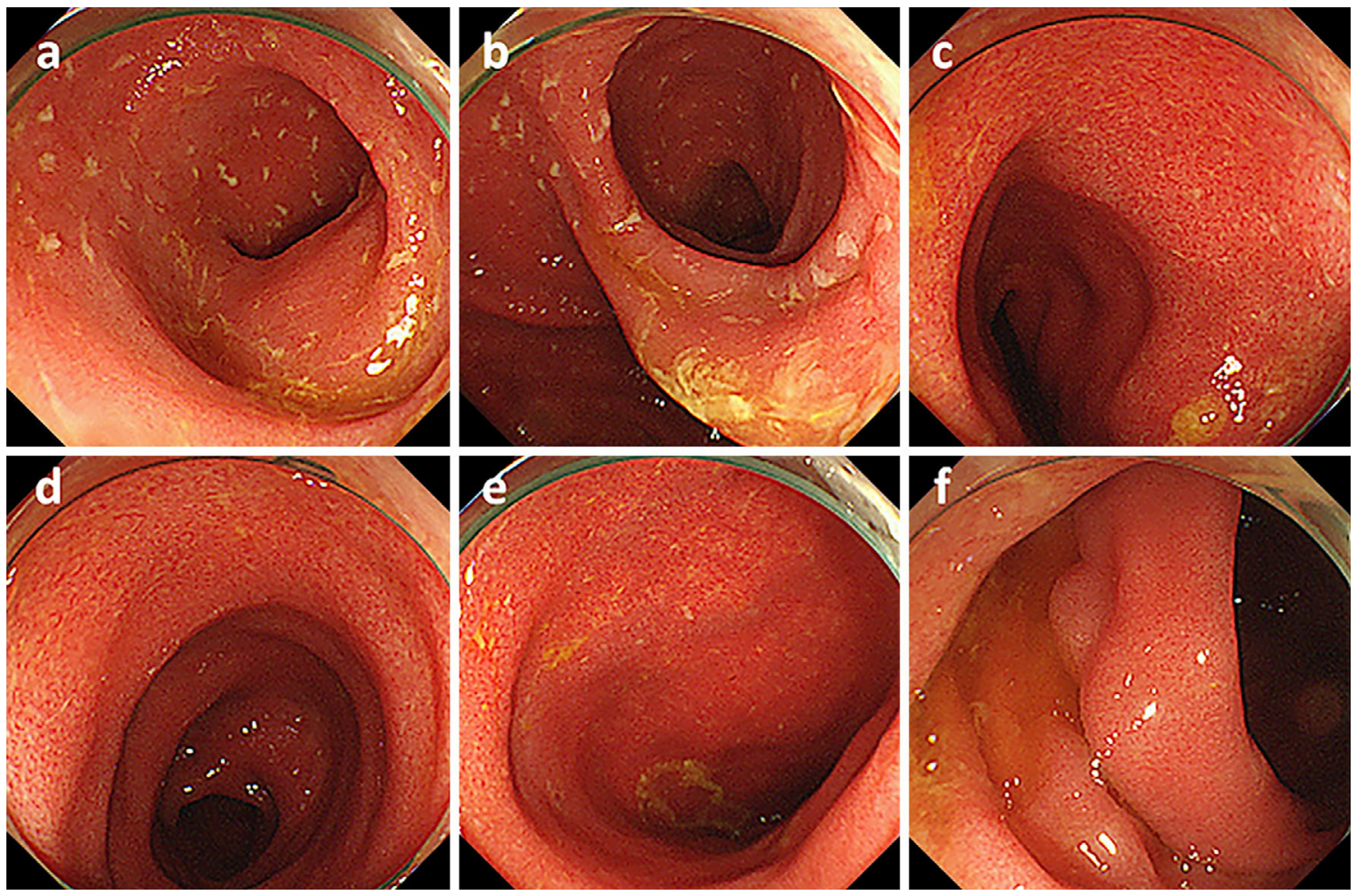

Figure 4. Colonoscopic findings at six months after time-dependent-release mesalazine was switched to the pH-dependent-release type at $3.6 \mathrm{~g} / \mathrm{d}$. Erosions were detected in the terminal ileum (a) and at the postoperative bowel anastomotic site (b). c: No improvement was observed in the findings of the large bowel (c: Transverse colon. d: Descending colon. e: Sigmoid colon. f: Rectum).

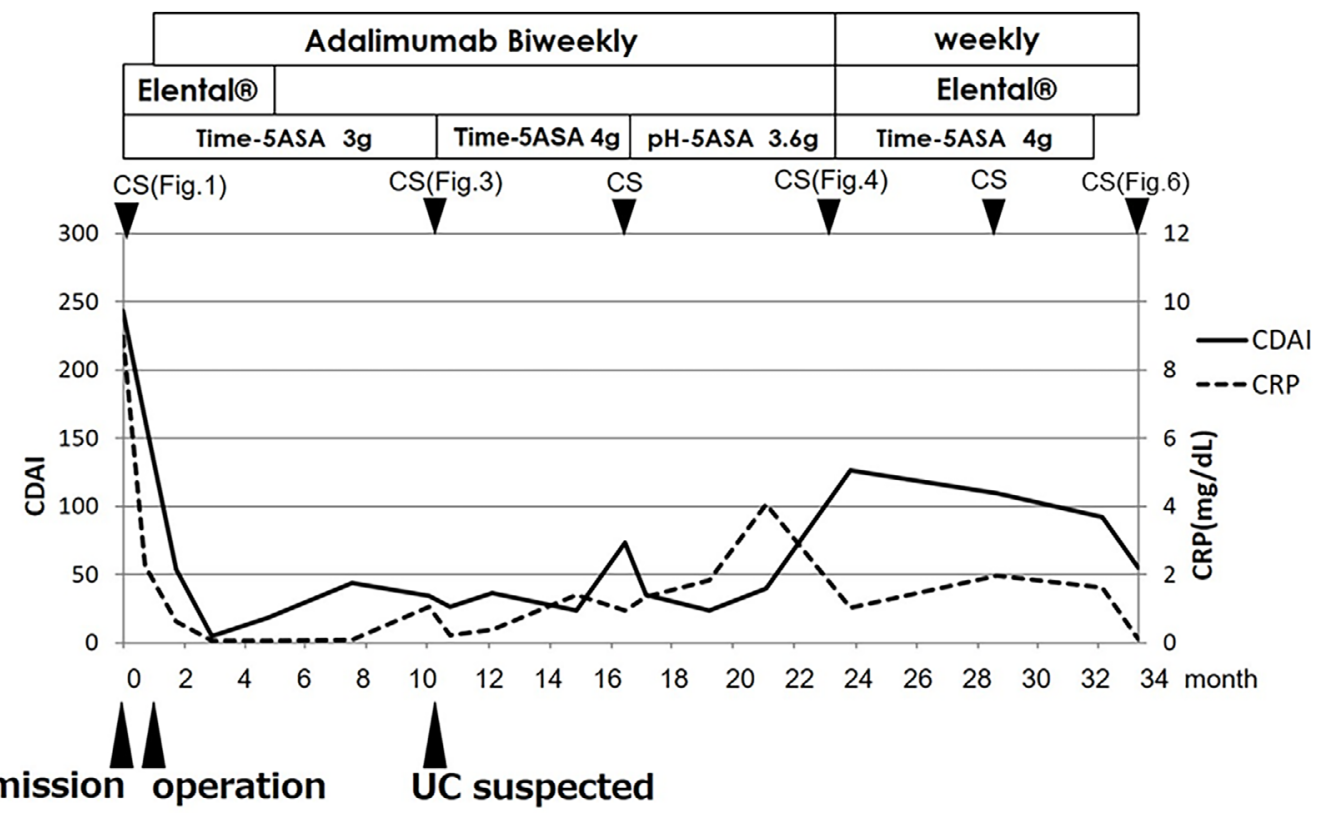

Figure 5. Clinical course.

classified (IBDU) complicating Crohn's disease-yielded any improvement. Given the atypical clinical course, we considered the possibility of mesalazine allergy. The results of the drug-induced lymphocyte stimulation test (DLST) were $1,119 \mathrm{cpm}$ with a stimulation index (SI) of $207 \%$ for timedependent-release mesalazine and $1,048 \mathrm{cpm}$ with a SI of $194 \%$ for $\mathrm{pH}$-dependent-release mesalazine. Thus, the DLST revealed positive reactions (SI >180) for both types of mesalazine. Therefore, the administration of mesalazine was discontinued, and by 1 month after the discontinuation of mesalazine, the CDAI had improved to 55, and the serum CRP level had decreased to $0.12 \mathrm{mg} / \mathrm{dL}$ (Fig. 5). Colonoscopy also revealed signs of improvement (Fig. 6). We were subsequently able to reduce the dose of adalimumab to 40 


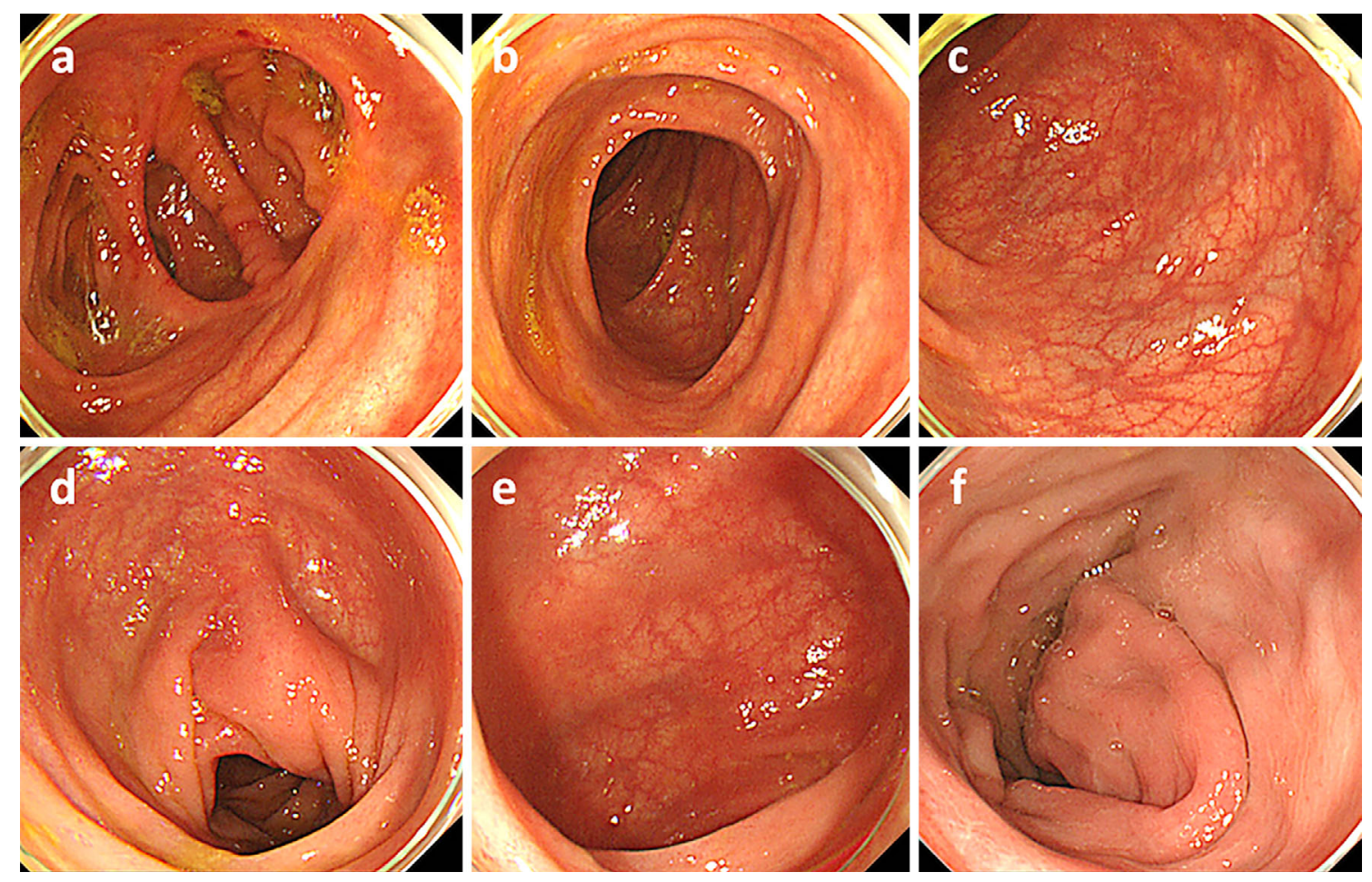

Figure 6. Colonoscopic findings at one month after the discontinuation of mesalazine. Improvement was observed in the findings of the large bowel (a: Postoperative bowel anastomotic site. b, $c$ : Transverse colon. d: Descending colon. e: Sigmoid colon. f: Rectum).

mg every 2 weeks. Thus, based on the clinical course described above, the postoperative exacerbation of the inflammatory findings on colonoscopy was considered to be attributable to mesalazine allergy.

\section{Discussion}

Mesalazine, a commonly used drug for treating both ulcerative colitis and Crohn's disease, can cause allergic reactions. The incidence of allergic reactions to mesalazine in patients with ulcerative colitis has been reported to range from $2.1 \%$ to $24 \%(3,4)$; however, that in patients with Crohn's disease remains unknown. There are few reports of allergic reactions to mesalazine in Crohn's disease patients. Although a DLST may be performed to complement the diagnosis of mesalazine allergy, as in our case, the sensitivity and specificity of a DLST in patients with ulcerative colitis are reportedly only $24 \%$ and $80.5 \%$, respectively (4). As such, the diagnosis should be mainly based on the clinical course.

In patients with mesalazine allergy, although the administration of mesalazine initially yields transient relief from clinical symptoms, patients often begin to suffer from diarrhea, exacerbation of bloody stool, or a fever relatively soon thereafter, typically within two weeks of starting treatment (4). However, if the symptomatic exacerbation is mistakenly diagnosed as being caused by exacerbation of the underlying inflammatory bowel disease and treated by the immediate addition of steroids, the symptoms will be masked. Then, when the symptoms relapse during tapering of the steroid dose, patients may be judged as having become steroid-dependent. Thus, they may be over-treated, or the diagnosis of mesalazine allergy may be delayed (5). In addition, the DLST detects type IV allergy, in which Tlymphocytes are involved, and adalimumab inhibits this type of allergic reaction. Thus, it is also plausible that in our case, adalimumab masked the mesalazine allergy and alleviated the symptoms. However, no association has been reported between adalimumab, an anti-tumor necrosis factor (TNF) $\alpha$ antibody, and mesalazine allergy, although there is a report of increased blood TNF- $\alpha$ levels in patients with multiple-drug hypersensitivity receiving adalimumab (6).

The two most likely reasons for the delay in the diagnosis of mesalazine allergy in our patient were as follows: First, the clinical symptoms and inflammatory findings may have transiently improved because right hemicolectomy was performed for circumferential strictures of the ascending colon on the 25th day of treatment with mesalazine, followed by the early initiation of treatment with adalimumab after surgery. Second, because the postoperative endoscopic findings resembled those of ulcerative colitis and a biopsy revealed the presence of crypt abscesses, we suspected comorbid ulcerative colitis or IBDU. Crypt abscess is a collection of neutrophils within the crypt lumen and is well-recognized as a histological feature of ulcerative colitis (7). Although crypt abscesses have not been described in cases of mesalazine allergy to date, they have been observed in other inflammatory diseases of the bowel, such as infectious enterocolitis (8) and diverticular colitis (9). Therefore, crypt abscesses are not unique to ulcerative colitis, so caution should be ex- 
ercised when encountering them.

In our case, we were able to longitudinally observe the changes in the bowel mucosa. Although there was no evidence of inflammation of the mucosa extending from the transverse colon to the rectum before the initiation of treatment with mesalazine, granularity of the mucosa with circumferential and continuous involvement from the transverse colon to the rectum, resembling the features of ulcerative colitis, was observed after the patient was started on mesalazine treatment. A previous case report of a patient with ulcerative colitis who underwent a challenge test with a mesalazine suppository stated that the endoscopic findings before administration of the suppository were only the loss of a visible vascular pattern and mild edema; in contrast, after the start of mesalazine administration, endoscopy revealed granular mucosa and contact bleeding, and a biopsy revealed diffuse neutrophilic infiltration up to the basement membrane and crypt epithelium, as well as mucus depletion (10).

Regarding Crohn's disease, there have been almost no reports of such subjects or of how the normal mucosal appearance in these patients changes with mesalazine allergy. Thus, the further accumulation of cases is necessary to determine whether or not there are any endoscopic findings specific to mesalazine allergy. Our experience with present case indicates that mesalazine allergy can manifest with endoscopic or histological findings resembling those of ulcerative colitis. In addition, our case showed diffuse aphthous ileitis in the terminal ileum and at the bowel anastomotic site after the time-dependent-release mesalazine was switched to $\mathrm{pH}$-dependent-release mesalazine. Because the effects of mesalazine allergy on the small intestine have not yet been reported, whether or not mesalazine allergy can affect the small intestine remains unclear. However, because the small intestinal findings were exacerbated after the timedependent-release mesalazine was switched to the $\mathrm{pH}$ dependent-release type under suspicion of comorbid ulcerative colitis, we assume that $\mathrm{pH}$-dependent-release mesalazine was ineffective, resulting in the exacerbation of the features of Crohn's disease in the terminal ileum and at the bowel anastomotic site.

If patients with positive DLST findings for mesalazine have only a mild allergic reaction, hyposensitization therapy may permit the continuous administration of mesalazine (11). Although we considered introducing hyposensitization therapy to this patient, we ultimately did not perform it because he achieved clinical and endoscopic remission with adalimumab treatment, and mesalazine as a maintenance treatment in Crohn's disease is no longer recommended under the European Crohn's and Colitis Organization (ECCO) guideline (12).

In conclusion, this case serves to highlight the need to consider the possibility of mesalazine allergy in patients with inflammatory bowel disease receiving the drug who show an atypical clinical course. We consider this case to be a valuable one, as we were able to longitudinally observe the development of the lesions caused by mesalazine allergy in previously normal mucosa. Because mesalazine allergy may manifest with endoscopic findings resembling those of ulcerative colitis, the further accumulation of cases in the future will be needed in order to determine the characteristic histological features of mesalazine allergy.

The authors state that they have no Conflict of Interest (COI).

\section{References}

1. Hanauer S, Schwartz J, Robinson M, et al. Mesalamine capsules for treatment of active ulcerative colitis: results of a controlled trial. Pentasa Study Group. Am J Gastroenterol 88: 1188-1197, 1993.

2. Gupta MK, Pollack S, Hutchings JJ. Mesalamine induced symptom exacerbation of ulcerative colitis: case report and brief discussion. World J Gastrointest Pharmacol Ther 1: 132-134, 2010.

3. Motoya S, Simodate Y, Tanaka H, Imamura A. Mesalamine intolerance of ulcerative colitis. IBD Research 4: 127-131, 2010 (in Japanese).

4. Saito D, Hayashida M, Sato T, et al. Evaluation of the druginduced lymphocyte stimulation test for diagnosing mesalazine allergy. Intest Res 16: 273-281, 2018.

5. Sturgeon JB, Bhatia P, Hermens D, Miner PB Jr. Exacerbation of chronic ulcerative colitis with mesalamine. Gastroenterology 108: 1889-1893, 1995.

6. Akhmaltdinova LL, Gazalieva MA, Akhmetova SB. Levels of cytokines in drug hypersensitivity. Cent Eur J Immunol 42: 354-357, 2017.

7. Gramlich T, Petras RE. Pathology of inflammatory bowel disease. Semin Pediatr Surg 16: 154-163, 2007.

8. Griffin PM, Olmstead LC, Petras RE. Escherichia coli O157:H7associated colitis. A clinical and histological study of 11 cases. Gastroenterology 99: 142-149, 1990.

9. Torii Y, Katano Y, Yoshino J, et al. A case of diverticular colitis with lesions resembling ulcerative colitis and correlation of tumor necrosis factor-alpha staining with clinical manifestations. Clin J Gastroenterol 8: 377-384, 2015.

10. Kapur KC, Williams GT, Allison MC. Mesalazine induced exacerbation of ulcerative colitis. Gut 37: 838-839, 1995.

11. Motoya S, Miyakawa M, Nasuno M, Tanaka H. Approach for mesalamine intolerance. IBD Research 9: 17-286, 2015 (in Japanese).

12. Fernando G, Axel D, Vito A, et al. 3rd European evidence-based consensus on the diagnosis and management of Crohn's disease 2016: Part 1: diagnosis and medical management. J Crohn's Colitis 11: $3-25,2017$.

The Internal Medicine is an Open Access journal distributed under the Creative Commons Attribution-NonCommercial-NoDerivatives 4.0 International License. To view the details of this license, please visit (https://creativecommons.org/licenses/ by-nc-nd/4.0/).

(C) 2019 The Japanese Society of Internal Medicine Intern Med 58: 649-654, 2019 